\title{
QUASI-EQUIVALENCE CLASSES OF NORMAL REPRESENTATIONS FOR A SEPARABLE $C^{*}$-ALGEBRA $\left({ }^{1}\right)$
}

BY

\author{
HERBERT HALPERN
}

\begin{abstract}
It is shown that the set of quasi-equivalence classes of normal representations of a separable $C^{*}$-algebra is a Borel subset of the quasi-dual with the Mackey Borel structure and forms a standard Borel space in the induced Borel structure. It is also shown that the set of factor states which induce normal representations forms a Borel set of the space of factor states with the $w^{*}$-topology and that this set has a Borel transversal.
\end{abstract}

Let $A$ be a separable $C^{*}$-algebra. Two representations $\lambda$ and $\lambda^{\prime}$ of $A$ on the Hilbert spaces $H(\lambda)$ and $H\left(\lambda^{\prime}\right)$ are said to be quasi-equivalent (in symbols: $\lambda \sim \lambda^{\prime}$ ) if there is an isomorphism $\Phi$ of the von Neumann algebra $\lambda(A)^{\prime \prime}$ generated by $\lambda(A)$ onto that generated by $\lambda^{\prime}(A)$ such that $\Phi(\lambda(x))=$ $\lambda^{\prime}(x)$ for every $x \in A$. A representation $\lambda$ of $A$ is a factor representation if the center of $\lambda(A)^{\prime \prime}$ consists of scalar multiples of the identity. The relation of quasi-equivalence partitions the factor representations of $A$ into quasi-equivalence classes. Let $\widetilde{A}$ denote the set of all quasi-equivalence classes of nonzero factor representations of $A$, and let $[\lambda]$ denote the quasi-equivalence class that contains the representation $\lambda$.

For any Hilbert space $H$, let $\operatorname{Rep}(A, H)$ (resp. $\operatorname{Fac}(A, H))$ denote the space of all representations (resp. factor representations) of $A$ on $H$ taken with the topology of pointwise convergence, i.e., $\lambda_{n} \rightarrow \lambda$ if and only if $\lambda_{n}(x) \zeta \rightarrow \lambda(x) \xi$ for all $x \in A$ and $\zeta \in H$. Let $H_{n}(n=1,2, \cdots, \infty)$ be a separable Hilbert space of dimension $n$, and let $\operatorname{Rep} A$ (resp. Fac $A$ ) be the disjoint union of the spaces $\operatorname{Rep}\left(A, H_{n}\right)\left(\operatorname{resp} . \operatorname{Fac}\left(A, H_{n}\right)\right)$ for $n=1,2, \cdots, \infty$. A subset $X$ of $\operatorname{Rep} A$ (resp. Fac $A$ ) is a Borel set if, for each $n$, the set $X \cap \operatorname{Rep}\left(A, H_{n}\right)$ (resp. $X \cap \operatorname{Fac}\left(A, H_{n}\right)$ ) is a Borel set in the Borel structure induced by the topology. The Borel space $\operatorname{Rep} A$ is standard in the sense that it is Borel isomorphic with a Borel subset of a polonais (i.e., a complete separable metrizable) space. (Note that a standard Borel space is determined up

Presented to the Society, September 24, 1973; received by the editors November 16, 1973.

AMS (MOS) subject classifications (1970). Primary 46L05.

Key words and phrases. Separable $C^{*}$-algebras, quasi-dual, normal representations, trace representations, factor states, standard Borel space.

$\left.{ }^{1}\right)$ This work was supported by the National Science Foundation. 
to Borel equivalence by its cardinality and only two infinite cardinals are possible [12, §3].) The set Fac $A$ is a Borel subset of $\operatorname{Rep} A$ and the Borel structure induced on Fac $A$ by $\operatorname{Rep} A$ is the structure already assigned to it. The map $\psi$ which assigns to each $\lambda$ its quasi-equivalence class $[\lambda]$ in $\widetilde{A}$ actually is surjective and induces the so-called Mackey Borel structure on $\widetilde{A}$, viz., a set $X$ is Borel in $\tilde{A}$ if and only if $\psi^{-1}(X)$ is Borel in Fac $A[6, \S \S 5,7]$.

A nondegenerate representation $\lambda$ of $A$ on the Hilbert space $H(\lambda)$ is said to be a trace representation of $A$ if the von Neumann algebra $\lambda(A)^{\prime \prime}$ generated by $\lambda(A)$ is semifinite and if there exists a faithful normal trace $t$ on $\lambda(A)^{\prime \prime+}$ such that $\lambda(A) \cap N(t)$ generates the von Neumann algebra $\lambda(A)^{\prime \prime}$. Here $N(t)$ is the ideal of definition of $t$ given by the set of all linear combinations of elements in the set

$$
N(t)^{+}=\left\{x \in \lambda(A)^{\prime \prime} \mid t(x)<+\infty\right\} .
$$

The set $N(t)$ is an ideal in $\lambda(A)$ " (cf. $[6, \S 6]$ ). (In the sequel a two-sided ideal closed under involution is simply called an ideal. Most of the ideals considered in this note will not be closed in the norm topology.)

If $\lambda$ is a trace representation of $A$ and if $\lambda(A)^{\prime \prime}$ is a factor von Neumann algebra, then $\lambda$ is called a normal representation of $\boldsymbol{A}$ [9, Definition, p. 13]. Since every trace representation gives a $\sigma$-finite von Neumann algebra $[6,6.3 .6]$, the faithful normal trace on $\lambda(A)^{\prime \prime+}$, where $\lambda$ is a normal representation, is unique up to a strictly positive scalar multiple $[7, \mathrm{I}, 6$, Theorem 4 , Corollary]. Thus, a semifinite factor representation $\lambda$ of $A$ is normal if and only if $\lambda(A)$ contains a nonzero element of finite trace. Indeed, if the ideal $\lambda(A) \cap N(t)$ is nonzero, then its weak closure is $\lambda(A)^{\prime \prime}[7, I, 3$, Theorem 2, Corollary 3].

In this note we show that the set $X$ of quasi-equivalence classes of normal representations of $A$ is a Borel subset of $\widetilde{A}$ and is standard in the induced Borel structure. This answers a question posed by J. Dixmier $[6,7.5 .4]\left({ }^{2}\right)$. We apply this to show that there is a Borel subset of factor states of $A$ (with the Borel structure induced by the $w^{*}$-topology) that is Borel isomorphic with $X$. A. Guichardet [9] proved that the quasi-equivalence classes of finite (resp. type I) normal representations is a Borel subset of $\widetilde{A}$ and is standard in the induced Borel structures. Other structures in this regard have been given by Perdrizet [14]. Although there are separable $C^{*}$-algebras with no normal representations [5], there are some (viz., the GCR algebras) for which every factor representation is normal and others (e.g., the reduced group $C^{*}$-algebra of a second countable, locally compact unimodular group $[9, I, \S 3$, Theorem 1 , Corollary $]$ ) such that, for every nonzero $x$ in the algebra, there is a normal representation $\lambda$ with $\lambda(x) \neq 0$.

$\left(^{2}\right)$ The rest of the problem has been solved by 0 . A. Nielsen. 
The first lemma is the basis of our analysis of the Borel structure.

Lemma 1. Let $A$ be a separable $C^{*}$-algebra. There is a countable subset $S$ of $A^{+}$such that, for every normal representation $\lambda$ of $A$, the set $\lambda(S)$ contains a nonzero element of finite trace.

Proof. Let $S_{1}$ be a countable dense subset of $\left\{x \in A^{+} \mid\|x\|=1\right\}$. Let $\alpha, \beta$ be rational numbers with $0<\alpha<\beta<1$ and let $f=f_{\alpha, \beta}$ be the continuous real-valued function of a real variable defined by $f(\gamma)=0$ if $\gamma \leqslant \alpha$, $f(\gamma)=1$ if $\gamma \geqslant \beta$, and $f$ linear on $[\alpha, \beta]$. Let $F$ be the (countable) family of functions $F=\left\{f_{\alpha, \beta} \mid \alpha, \beta\right.$ rational and $\left.0<\alpha<\beta<1\right\}$. Let $S$ be the countable subset of $A^{+}$given by $S=\left\{f(x) \mid f \in F, x \in S_{1}\right\}$.

Let $\lambda$ be a normal representation of $A$ and let $t$ be a faithful, normal, semifinite trace on $\lambda(A)^{\prime \prime+}$. We show that there is an $x \in S$ such that $0<$ $t \lambda(x))<+\infty$. Let $J$ be the closed ideal of $\lambda(A)^{\prime \prime}$ generated by the finite projections of $\lambda(A)^{\prime \prime}$ (cf. [10, §2]). Let $y$ be a nonzero element in $\lambda(A) \cap$ $N(t)$. Since $y^{*} y \in \lambda(A) \cap N(t)$, we may assume that $y \in \lambda(A)^{+}$. Let $0<\alpha<$ $\|y\|$ and let $e$ be the spectral projection of $y$ (in $\left.\lambda(A)^{\prime \prime}\right)$ corresponding to the interval $[\alpha,\|y\|]$. We have that $e \leqslant \alpha^{-1} y$ and thus that $e$ has finite trace. This proves that $e \in J$. We also have that $\|y-e y\| \leqslant \alpha$. Because $\alpha>0$ may be arbitrarily small, we get that $y \in J$. This means that the closed ideal $\lambda(A) \cap J$ of $\lambda(A)$ is not zero, and therefore, the canonical homomorphism $\phi$ of the $C^{*}$. algebra $\lambda(A)$ onto the $C^{*}$-algebra $\lambda(A) / \lambda(A) \cap J$ is not an isometry. But if

$$
\|\lambda(z)\|=\operatorname{glb}\{\|\lambda(z)+w\| \mid w \in J \cap \lambda(A)\}=\|\phi(\lambda(z))\|
$$

for every $z \in S_{1}$, then $\|z\|=\|\phi(z)\|$ for every $z$ in the unit sphere of $\lambda(A)$ due to the continuity of the maps $z \rightarrow\|\lambda(z)\|$ and $z \rightarrow\|\phi(\lambda(z))\|$ on $A$. This means that the canonical homomorphism $\phi$ is an isometry. Therefore, we may find a $z \in S_{1}$ and an $\alpha$ with $0<\alpha<1 / 2$ such that

$$
\|\phi(\lambda(z))\|<(1-2 \alpha)\|\lambda(z)\|<\|\lambda(z)\| \leqslant 1 .
$$

Let $e^{\prime}$ be the spectral projection of $\lambda(z)$ corresponding to the interval $[(1-\alpha)\|\lambda(z)\|,\|\lambda(z)\|]$. We have that

$$
\lambda(z) \geqslant(1-\alpha)\|\lambda(z)\| e^{\prime}
$$

and thus that

$$
\lambda(z)(\bmod J) \geqslant(1-\alpha)\|\lambda(z)\| e^{\prime}(\bmod J) \geqslant 0
$$

in the $C^{*}$-algebra $\lambda(A)^{\prime \prime} / J$. We get that 


$$
(1-2 \alpha)\|\lambda(z)\| \geqslant\|\phi(\lambda(z))\|=\|\lambda(z)(\bmod J)\| \geqslant(1-\alpha)\|\lambda(z)\|\left\|e^{\prime}(\bmod J)\right\| .
$$

We find the norm of the projection $e^{\prime}(\bmod J)$ is zero since the only possible choices for its norm are 0 and 1 . Hence the projection $e^{\prime}$ is in $J$. We recall that all the projections in $J$ are finite projections [10, Proposition 2.1]. Now we may find a function $f$ in $F$ such that $f(\lambda(z)) \neq 0$ and such that $f(\lambda(z)) \leqslant e^{\prime}$. For example, let $f=f_{\beta, \gamma}$ where $\beta$ and $\gamma$ are rational numbers that satisfy $(1-\alpha)\|\lambda(z)\|<\beta<\gamma<\|\lambda(z)\|$. Since $f(\lambda(z))=\lambda(f(z))$, the element $x=f(z)$ in $S$ is not in the kernel of $\lambda$ and satisfies the relation $0 \leqslant \lambda(x) \leqslant e^{\prime}$. This proves that $\lambda(x)$ is a nonzero element of finite trace. Q.E.D.

Let $I$ be an ideal of the $C^{*}$-algebra $A$. A complex-valued function $s$ of the cartesian product $I \times I$ is called a bitrace with ideal of definition $I$ if $s$ satisfies the following axioms: (i) $s$ is a positive hermitian form on $I \times I$; (ii) $s(x, y)=s\left(y^{*}, x^{*}\right)$, for all $x, y \in I$; (iii) $s(z x, y)=s\left(x, z^{*} y\right)$, for all $x, y \in I$ and $z \in A$; (iv) for every $x \in A$, the map $z \rightarrow x z$ defines a continuous linear operator of $I$ into $I$ in the prehilbert structure induced by $s$; and (v) the set $I^{2}=\{x y \mid x, y \in I\}$ is dense in $I$ in the prehilbert structure induced by $s$ [4]. Every bitrace $s$ with ideal of definition I induces a trace representation of $A$ in a canonical way. In fact, first assume that $s$ satisfies only the properties (i)(iii). Let $\Lambda_{s}$ be the canonical homomorphism of $I$ into $I$ modulo the ideal $\{x \in I \mid s(x, x)=0\}$. For every $x, y \in I$, the relation $\left(\Lambda_{s}(x), \Lambda_{s}(y)\right)=s(x, y)$ defines an inner product on $\Lambda_{s}(I)$. Let $H_{s}$ be the completion of $\Lambda_{s}(I)$ in this inner product. Now assume $s$ satisfies property (iv). For every $x \in A$ the map $\Delta_{s}(y) \rightarrow \Lambda_{s}(x y)$ (resp. $\Lambda_{s}(y) \rightarrow \Lambda_{s}(y x)$ ) of $\Lambda_{s}(I)$ can be extended to a bounded linear operator $\lambda_{s}(x)$ (resp. $\rho_{s}(x)$ ) of the Hilbert space $H_{s}$, and the map $x \rightarrow \lambda_{s}(x)$ (resp. $x \rightarrow \rho_{s}(x)$ ) defines a representation (resp. antirepresentation) of $A$ on $H_{s}$. This means that $\left\|\lambda_{s}(x)\right\| \leqslant\|x\|$ (resp. $\left.\left\|\rho_{s}(x)\right\| \leqslant\|x\|\right)$ since every representation of $A$ is norm decreasing. Suppose now that $s$ satisfies property (v). We then have that $\lambda_{s}(A)^{\prime \prime}=\rho_{s}(A)^{\prime}$. Furthermore, there is a faithful normal semifinite trace $t$ on $\lambda_{s}(A)^{\prime \prime+}$ such that $\left.t \lambda_{s}\left(x x^{*}\right)\right)=s(x, x)$ for all $x \in I$ and such that $\lambda(A) \cap N(t)$ generates the von Neumann algebra $\lambda(A)^{\prime \prime}([4],[9]$, cf. $[6,6.2])$.

Conversely, let $\lambda$ be a trace representation of $A$ on the Hilbert space $H$. Let $t$ be a faithful normal semifinite trace on $\lambda(A)^{\prime \prime}$ such that $\lambda(A) \cap N(t)$ generates $\lambda(A)^{\prime \prime}$. The set $\left.I=\left\{x \in A \mid t \lambda(x) \lambda(x)^{*}\right)<+\infty\right\}$ is an ideal in $A$ and the relation $s(x, y)=t^{\prime}\left(\lambda\left(x y^{*}\right)\right)$ defines a bitrace with ideal of definition $I$. Here $t^{\prime}$ is the unique extension of $t$ to a linear functional on $N(t)$. The canonical representation $\lambda_{s}$ induced by $s$ is quasi-equivalent to $\lambda[6,6.6 .5$ (ii)] 
Proposition 2. Let $x_{0} \in A^{+}$, let $I=I\left(x_{0}\right)$ be the ideal of $A$ generated by $x_{0}$, and let $T=T\left(x_{0}\right)$ be the family of all bitraces on $I$ such that $s\left(x_{0}, x_{0}\right)=1$; then in the topology of pointwise convergence on $I \times I$, the space $T$ is polonais.

Proof. Let $T^{\prime}$ be the set of all complex-valued functions $s$ on $I \times I$ satisfying properties (i)-(iv) of the definition of bitraces and the additional property (vi) $s\left(x_{0}, x_{0}\right)=1$. Notice that $T$ is a subset of $T^{\prime}$.

Let $A_{e}$ be the $C^{*}$-algebra $A$ if $A$ has identity or the $C^{*}$-algebra $A$ with identity $e_{0}$ adjoined (cf. $[6,1.3 .8]$ ) if $A$ has no identity. If $A$ has no identity, the map $(x, \alpha) \rightarrow x+\alpha e_{0}$ of the Banach space given by the cartesian product of $A$ with the complexes with norm $\|(x, \alpha)\|=\|x\|+|\alpha|$ onto the $C^{*}$-algebra $A_{e}$ with norm $\left\|x+\alpha e_{0}\right\|=\operatorname{lub}\{\|x y+\alpha y\| \mid y \in A,\|y\| \leqslant 1\}$ is continuous and one-one. Therefore, the inverse of the map is continuous, and so there is a constant $k \geqslant 1$ with $\|x\|+|\alpha| \leqslant \kappa\left\|x+\alpha e_{0}\right\|$. Using $A_{e}$, we can explicitly express the ideal $I$ as

$$
I=\left\{\sum\left\{x_{i} x_{0} y_{i} \mid 1 \leqslant i \leqslant m\right\} \mid x_{i}, y_{i} \in A_{e}, m=1,2, \cdots\right\} .
$$

For each $x, y \in I$, we show that the set $\left\{|s(x, y)| \mid s \in T^{\prime}\right\}$ is bounded. Let $s \in T^{\prime}$; then, for $x, y \in A_{e}$, we get by direct calculation that

$$
s\left(x x_{0} y, x x_{0} y\right) \leqslant \kappa^{4}\|x\|^{2}\|y\|^{2},
$$

and thus, for $x_{i}, y_{i}(1 \leqslant i \leqslant m), x_{i}^{\prime}, y_{i}^{\prime}(1 \leqslant i \leqslant n)$ in $A_{e}$, we get that

$$
s\left(\sum x_{i} x_{0} y_{i}, \sum x_{i}^{\prime} x_{0} y_{i}^{\prime}\right) \leqslant \sum_{i, j} s\left(x_{i} x_{0} y_{i}, x_{i} x_{0} y_{i}\right)^{1 / 2} s\left(x_{j}^{\prime} x_{0} y_{j}^{\prime}, x_{j}^{\prime} x_{0} y_{j}^{\prime}\right)^{1 / 2}
$$

$$
\leqslant \sum_{i, j} \kappa^{4}\left\|x_{i}\right\|\left\|x_{j}^{\prime}\right\|\left\|y_{i}\right\|\left\|y_{j}^{\prime}\right\| .
$$

Setting

$$
\alpha\left(\sum x_{i} x_{0} y_{i}, \sum x_{i}^{\prime} x_{0} y_{i}^{\prime}\right)=\sum \kappa^{4}\left\|x_{i}\right\|\left\|x_{j}^{\prime}\right\|\left\|y_{i}\right\|\left\|y_{j}^{\prime}\right\|,
$$

we obtain a positive real-valued function on $I \times I$ that is independent of the choice of $s$ in $T^{\prime}$.

It is now possible to define a metric on $T^{\prime}$. Let $B$ be a countable dense subset of $A_{e}$ containing the identity. Let $\left\{u_{i}\right\}$ be an enumeration of the countable dense subset of $I$

$$
C=\left\{\sum\left\{x_{i} x_{0} y_{i} \mid 1 \leqslant i \leqslant m\right\} \mid x_{i}, y_{i} \in B, m=1,2, \cdots\right\},
$$


and let $d$ be the positive real-valued function of $T^{\prime} \times T^{\prime}$ given by

$$
d(r, s)=\sum_{i, j}\left|r\left(u_{i}, u_{j}\right)-s\left(u_{i}, u_{j}\right)\right| / 2^{i+j}\left(\alpha\left(u_{i}, u_{j}\right)+1\right) .
$$

Due to the bound $\left|s\left(u_{i}, u_{j}\right)\right| \leqslant \alpha\left(u_{i}, u_{j}\right)$ on $s$, the function $d$ is finite-valued. To verify that $d$ is a metric it is necessary to verify $d(r, s)=0$ implies $r=s$; the other properties of a metric are clearly satisfied. Let $d(r, s)=0$. Let $x_{i}$, $x_{i}^{\prime} \in A_{e}$ and let $b_{i}, b_{i}^{\prime} \in B$ for $1 \leqslant i \leqslant m$; then, for every $p \in T^{\prime}$, the elements $\Lambda_{p}\left(\Sigma b_{i} x_{0} b_{i}^{\prime}\right)$ tend to $\Lambda_{p}\left(\Sigma x_{i} x_{0} x_{i}^{\prime}\right)$ in $H_{p}$ as the $b_{i}$ and $b_{i}^{\prime}$ tend to the $x_{i}$ and $x_{i}^{\prime}$ respectively due to the continuity of the functions $x, y \rightarrow \Lambda_{p}(x z y)$ on $A_{e} \times A_{e}$, for fixed $z \in I$ (cf. (1)). (Note that this means that $\Lambda_{p}(C)$ is dense in $\Lambda_{p}(I)$.) In particular, we get that

$$
\left(\Lambda_{p}\left(\sum b_{i} x_{0} b_{i}^{\prime}\right), \Lambda_{p}\left(\sum b_{i} x_{0} b_{i}^{\prime}\right)\right) \rightarrow\left(\Lambda_{p}\left(\sum x_{i} x_{0} x_{i}^{\prime}\right), \Lambda_{p}\left(\sum x_{i} x_{0} x_{i}^{\prime}\right)\right)
$$

as $b_{i} \rightarrow x_{i}$ and $b_{i}^{\prime} \rightarrow x_{i}^{\prime}$ for all $i$. This implies that $r(x, x)=s(x, x)$ for all $x \in I$ and consequently that $r=s$ by polarization.

We now show that the metric topology on $T^{\prime}$ is the same as the topology of pointwise convergence. In fact, let $\left\{s_{n}\right\}$ be a net on $T^{\prime}$ that converges to $s$ in $T^{\prime}$ in the metric or equivalently, pointwise on $C \times C$. But given $x \in I$ and $\epsilon>0$, there is a $u \in C$ such that $|r(u, u)-r(x, x)|<\epsilon$ for all $r \in T^{\prime}$. This implies that $\lim s_{n}(x, x)=s(x, x)$ for all $x \in I$, and thus, that $\left\{s_{n}\right\}$ converges to $s$ pointwise on $I$.

Now, in the usual way, we can identify $T^{\prime}$ with a closed subspace of the product of compact subsets of the complex numbers. Let $\Pi$ be the compact space given by

$$
\Pi=\prod\{\{\alpha \text { complex }|| \alpha \mid \leqslant \alpha(x, y)\} \mid x, y \in \Pi\},
$$

and let $\Phi$ be the homeomorphism of $T^{\prime}$ into $\Pi$ given by $\Phi(s)_{x, y}=s(x, y)$. Let $\left\{s_{n}\right\}$ be a net in $T^{\prime}$ such that $\left\{\Phi\left(s_{n}\right)\right\}$ converges to $r$ in $\Pi$. Setting $r_{x, y}=s(x, y)$, we obtain a complex-valued function of $I \times I$ that satisfies properties (i)-(iii) of the definition of a bitrace. For $x \in A, y \in I$, we have that

$$
s(x y, x y)=\lim s_{n}(x y, x y) \leqslant\|x\|^{2} \lim \sup s_{n}(y, y)=\|x\|^{2} s(y, y) .
$$

This means that $s$ satisfies property (iv). Also we see that $\Phi(s)=r$. Hence, we get that $\Phi\left(T^{\prime}\right)$ is closed in $\Pi$, and so we have that $T^{\prime}$ is compact.

We can finish the proof by showing that $T$ is a $G_{\delta}$ in $T^{\prime}$ since every $G_{\delta}$ in a complete metric space is complete and metrizable in the induced topology 
[2, §6, Propositions 2 and 3]. Let $\left\{u_{i}^{\prime}\right\}$ be an enumeration of the countable set $\left\{u_{i} u_{j} \mid i, j=1,2, \cdots\right\}$. For every triple $i, j, k$ of integers, let $X_{i j k}$ be the open subset of $T^{\prime}$ given by

$$
X_{i j k}=\left\{s \in T^{\prime} \mid s\left(u_{i}^{\prime}-u_{j}, u_{i}^{\prime}-u_{j}\right)<k^{-1}\right\} .
$$

Then $T$ can be written as the $G_{\delta}$-set $X=\bigcap_{j, k} \cup_{i} X_{i j k}$. In fact, if $s \in X$, then $s$ satisfies (v) since $\Lambda_{s}(C)$ is dense in $I$ and since $\Lambda_{s}\left(C^{2}\right) \subset \Lambda_{s}\left(I^{2}\right)$. The converse relation is known (cf. [9, I, §1, Remark 4]). Q.E.D.

We now can prove the main result.

TheOREM 3. Let $A$ be a separable $C^{*}$-algebra; then the set of quasiequivalence classes of normal representations of $A$ is a Borel set in the quasidual of $A$ with the Mackey Borel structure and is standard in the induced Borel structure.

Proof. Let $S$ be a countable subset of $A^{+}$such that, for every normal representation $\lambda$ of $A$, the set $\lambda(S)$ contains a nonzero element of finite trace (Lemma 1). For each $x$ in $S$, let $I(x)$ be the ideal generated by $x$ and let $T(x)$ be the set of all bitraces $s$ on $I(x)$ such that $s(x, x)=1$. There is a Borel map $\phi=\phi_{x}$ of the family of all bitraces $s$ on $I(x) \times I(x)$ taken with the Borel structure induced by the topology of pointwise convergence on $I(x) \times$ $I(x)$ into $\operatorname{Rep} A$ such that $\phi(s)$ is quasi-equivalent to $\lambda_{s}$ [9, Chapter I, 2 , Lemma 2]. Because the set $T(x)$ and the inverse image $\phi^{-1}(\mathrm{Fac} A)$ under the Borel map $\phi$ are certainly Borel sets in the family of all bitraces on $I(x) \times$ $I(x)$ and because the Borel structure induced on $T(x)$ by the family of all bitraces coincides with that already assigned to $T(x)$, the restriction $\theta=\theta_{x}$ of $\phi$ to the Borel subset $T=T_{x}=T(x) \cap \phi^{-1}(\mathrm{Fac} A)$ of $T(x)$ is certainly a one-one Borel map of the Standard Borel space $T$ into Fac $A$. The fact that $T$ is standard follows from the fact that it is a Borel subset of the polonais space $T(x)$ (Proposition 2). We now verify that $\theta$ is one-one. In fact, we show more: If $r, s$ are in $T$ and if $\theta(r)$ and $\theta(s)$ are quasi-equivalent, then $r=s$. Indeed, let $\theta(r) \sim \theta(s)$. Since $\lambda_{r} \sim \theta(r)$ and $\lambda_{s} \sim \theta(s)$, there is an isomorphism $\Phi$ of $\lambda_{s}(A)^{\prime \prime}$ onto $\lambda_{r}(A)^{\prime \prime}$ such that $\Phi\left(\lambda_{s}(y)\right)=\Phi\left(\lambda_{r}(y)\right)$ for all $y \in A$. Let $t_{r}$ and $t_{s}$ be faithful normal semifinite traces on $\lambda_{r}(A)^{\prime \prime}$ and $\lambda_{s}(A)^{\prime \prime}$ respectively such that

$$
t_{r}\left(\lambda_{r}\left(y y^{*}\right)\right)=r(y, y) \text { and } t_{s}\left(\lambda_{s}\left(y y^{*}\right)\right)=s(y, y) \text {, }
$$

for all $y \in I(x)$. The function $t_{r} \cdot \Phi$ defines a faithful, normal, semifinite trace on $\lambda_{s}(A)^{\prime \prime}$ because $\Phi$ preserves least upper bounds of monotonely increasing 
nets in $\lambda_{s}(A)^{\prime \prime+}$. Since the trace of $\lambda_{s}(A)^{\prime \prime}$ is unique up to a strictly positive scalar multiple, there is an $\alpha>0$ such that $t_{r} \cdot \Phi=\alpha t_{s}$. But we have that

$$
\begin{aligned}
1 & =r(x, x)=t_{r}\left(\lambda_{r}\left(x x^{*}\right)\right)=t_{r}\left(\Phi\left(\lambda_{s}\left(x x^{*}\right)\right)\right) \\
& =\alpha t_{s}\left(\lambda_{s}\left(x x^{*}\right)\right)=\alpha s(x, x)=\alpha .
\end{aligned}
$$

Hence $\alpha=1$, and so $t_{r} \cdot \Phi=t_{s}$. Thus for all $y \in I(x)$, we have that

$$
r(y, y)=t_{r}\left(\lambda_{r}\left(y y^{*}\right)\right)=t_{s}\left(\lambda_{s}\left(y y^{*}\right)\right)=s(y, y) .
$$

This proves that $r=s$. Now $\theta$ is a one-one Borel function of the standard Borel space $T$ into the standard Borel space Fac $A$. Thus, the image $\theta(T)$ of $T$ is a Borel subset of Fac $A$ and the map $\theta$ is a Borel isomorphism of $T$ onto $\theta(T)$ [1, Proposition 2.5]. Let $\psi$ be the mapping of Fac $A$ onto $\tilde{A}$ which associates with each element $\lambda$ of Fac $A$ its quasi-equivalence class $[\lambda]$. Since the Borel set $\theta(T)$ of Fac $A$ meets each quasiequivalence class in at most one point, the image $\psi(\theta(T))$ of $\theta(T)$ is a Borel set in $\widetilde{A}$ and $\psi$ is a Borel isomorphism of $\theta(T)$ onto $\psi(\theta(T))[6,7.2 .3]$. Hence, the set $\psi(\theta(T))$ is a Borel subset of $\widetilde{A}$ and a standard Borel space in the induced Borel structure. Thus we get that $X=\left\{\psi\left(\theta_{x}\left(T_{x}\right)\right) \mid x \in S\right\}$ is a Borel subset of $\widetilde{A}$ since $S$ is countable, and that $X$ is a standard Borel space since $X$ may be written as a disjoint countable union of Borel subsets of the $\psi\left(\theta_{x}\left(T_{x}\right)\right)$ and such Borel subsets as well as disjoint countable unions of standard Borel spaces are standard [12, Theorem 3.1 and Theorem 3.2, Corollary 1].

We finish the proof by showing that $X$ contains every quasi-equivalence class of normal representations for $A$. Let $\lambda$ be a normal representation of $A$ and let $t$ be a faithful normal semifinite trace of $\lambda(A)^{\prime \prime}$. There is an element $x \in S$ such that $0<t\left(\lambda(x)\right.$ ) $<+\infty$ (Lemma 1). Since $\lambda(x) \in \lambda(A)^{+}$we have that

$$
0<t \lambda(x) \lambda(x)) \leqslant\|\lambda(x)\| t(\lambda(x))<+\infty .
$$

There is no loss in generality in the assumption that $t(\lambda(x) \lambda(x))=1$. We may define a bitrace $r$ on the ideal

$$
\left.I=\left\{y \in A \mid t \lambda(y) \lambda(y)^{*}\right)<+\infty\right\}
$$

by setting $r(y, z)=t^{\prime}\left(\lambda(y) \lambda(z)^{*}\right)$ for all $y, z \in I$. Here $t^{\prime}$ is the unique extension of $t$ to a linear functional on its ideal of definition. The canonical representation $\lambda_{r}$ induced by $r$ is quasi-equivalent to $\lambda$ (cf. introductory remarks, Proposition 2). Because $x \in I$, we get that $I(x) \subset I$. Let $s$ be the restriction of $r$ to $I(x) \times I(x)$. It is clear that $s$ satisfies properties (i)-(iv) in the 
definition of a bitrace on $I(x) \times I(x)$ plus the property (vi) $s(x, x)=1$. We show that $s \in T(x)$ by showing that $\Lambda_{s}\left((I(x))^{2}\right)$ is dense in $\Lambda_{s}(I(x))$. Let $\left\{x_{n}\right\}$ be an increasing approximate identity for $I(x)$ in the positive part of the unit sphere $I(x)[6,1.7 .2]$. For every $y \in I(x)$, we have from (1) that

$$
\begin{aligned}
s\left(\left(1-x_{n}\right) y,\left(1^{\circ}-x_{n}\right) y\right) & \leqslant \kappa^{4}\left\|1-x_{n}\right\| t^{\prime}\left(\lambda\left(\left(1-x_{n}\right) y\right) \lambda(y)^{*}\right) \\
& \leqslant \kappa^{4} t^{\prime}\left(\lambda\left(\left(1-x_{n}\right) y\right) \lambda(y)^{*}\right) .
\end{aligned}
$$

Because the function $z \rightarrow t^{\prime}\left(z \lambda(y)^{*}\right)$ is continuous on $\lambda(A)^{\prime \prime} \quad$ [7, I, §6, Proposition 1], we conclude that

$$
\lim s\left(\left(1-x_{n}\right) y,\left(1-x_{n}\right) y\right)=0 .
$$

This proves that $\Lambda_{s}\left(I(x)^{2}\right)$ is dense in $\Lambda_{s}(I(x))$. Therefore the function $s$ is in the set $T(x)$. We now show that the canonical representation $\lambda_{s}$ is unitarily equivalent to $\lambda_{r}$. Because $\lambda_{r} \sim \lambda$, this would imply on the one hand that $\lambda_{s}$ is a factor representation and therefore that $s \in T_{x}$. On the other hand, this would imply $\theta_{x}(s) \sim \lambda_{s} \sim \lambda_{r} \sim \lambda$, and consequently, we would get $[\lambda] \in$ $\psi\left(\theta_{x}\left(T_{x}\right)\right)$. Hence the set $X$ would contain all quasi-equivalence classes of normal representations. We proceed with the proof that $\lambda_{s}$ is unitarily equivalent to $\lambda_{r}$. We have that the linear manifold $\Lambda_{r}(I(x))$ in $H_{r}$ is invariant under $\lambda_{r}(A)$ and $\rho_{r}(A)$. This means that the closure of $\Lambda_{r}(I(x))$ in $H_{r}$ corresponds to a projection $e$ in $\lambda_{r}(A)^{\prime} \cap \rho_{r}(A)^{\prime}=\lambda_{r}(A)^{\prime} \cap \lambda_{r}(A)^{\prime \prime}$. Because $\lambda_{r}(A)^{\prime \prime}$ is a factor von Neumann algebra and because $e \Lambda_{r}(x)=\Lambda_{r}(x) \neq 0$, the projection $e$ is equal to the identity, or equivalently, $\Lambda_{r}(I(x))$ is dense in $H_{r}$. This means that the map $\Lambda_{s}(y) \rightarrow \Lambda_{r}(y)$ of $\Lambda_{s}(I(x))$ onto $\Lambda_{r}(I(x))$ can be extended to an isometric isomorphism $u$ of $H_{s}$ onto $H_{r}$. For every $y \in A, z \in I(x)$, we get that

$$
u \lambda_{s}(y) u^{-1} \Lambda_{r}(z)=u \Lambda_{s}(y x)=\Lambda_{r}(y z)=\lambda_{r}(y) \Lambda_{r}(z) .
$$

Consequently the representations $\lambda_{r}$ and $\lambda_{s}$ are unitarily equivalent via $u$. Q.E.D. A measure $\mu$ on a Borel space $X$ is said to be standard if there is a $\mu$-null Borel subset $M$ of $X$ such that $X-M$ is standard in the induced Borel structure. Decomposition theorems for traces and trace representations are formulated in terms of standard measures confined almost everywhere to the quasiequivalence class of normal representations (cf. [3], [6], [9], [13]). This is seen to be unnecessary.

COROLlary 4. The set of Borel measures of the quasi-equivalence classes of normal representations of a separable $C^{*}$-algebra (with the Mackey Borel structure) coincides with the set of standard Borel measures. 
Let $f$ be a state of the $C^{*}$-algebra $A$ (i.e., of positive linear functional on $A$ of norm 1); let $L_{f}$ be the so-called left kernel of $f$ given by

$$
L_{f}=\left\{x \in A \mid f\left(x^{*} x\right)=0\right\} .
$$

The set $L_{f}$ is a closed left ideal. Let $L_{f}(x)$ denote the image of $x$ in $A$ under the canonical homomorphism of $A$ into $A\left(\bmod L_{f}\right)$. The relation

$$
\left(L_{f}(x), L_{f}(y)\right)=f\left(y^{*} x\right)
$$

defines an inner product on $A\left(\bmod L_{f}\right)$. Let $H_{f}$ denote the completion of $A\left(\bmod L_{f}\right)$. If $x \in A$, the map $L_{f}(y) \rightarrow L_{f}(x y)$ can be extended to a bounded linear operator $\lambda_{f}(x)$ of the Hilbert space $H_{f}$. The map $x \rightarrow \lambda_{f}(x)$ is a representation of $A$ called the canonical representation induced by $f$ (cf. $[6,2.4 \mathrm{ff}$.$] ). A state f$ is called a factor state if $\lambda_{f}$ is a factor representation of $A$. Let $F(A)$ be the space of factor states of $A$ with the relativized $w^{*}$-topology. The space $F(A)$ is a standard Borel space with the Borel structure induced by the topology $([15,3.4 .5]$ and $[11$, Lemma 7$])$. Two elements $f$ and $g$ of $F(A)$ are said to be quasi-equivalent (in symbols: $f \sim g$ ) if $\lambda_{f} \sim \lambda_{g}$. The relation of quasi-equivalence partitions $F(A)$ into quasi-equivalence classes. The map $\psi_{1}(f)=\left[\lambda_{f}\right]$ maps $F(A)$ onto $\widetilde{A}$. A set $X$ in $F(A)$ is said to be saturated for the relation of quasi-equivalence if $g \in X$ whenever $g \sim f$ for some $f \in X$. A subset $X_{0}$ of the set $X$ in $F(A)$ is said to be a transversal of $X$ if, for each $f \in X$, the set $X_{0}$ meets $\psi_{1}^{-1}\left(\left[\lambda_{f}\right]\right)$ in exactly one point.

THEOREM 5. Let $A$ be a separable $C^{*}$-algebra, let $F(A)$ be the factor states of $A$, and let $X$ be the set of all factor states whose canonical representations are normal. Then the set $X$ is a saturated Borel set of $F(A)$ with a Borel transversal.

Proof. Let $\psi_{1}$ be the map of $F(A)$ onto $\widetilde{A}$ given by $\psi_{1}(f)=$ $\left[\lambda_{f}\right]$. A subset $Y$ of $\tilde{A}$ is a Borel subset of $\widetilde{A}$ if and only if $\psi_{1}^{-1}(Y)$ is a Borel subset of $F(A)$ [11, Theorem 8]. Using this fact and Theorem 3, we conclude that the set $X$ is a saturated Borel set of $F(A)$.

Let $S$ be a countable dense subset of $A^{+}$such that, for every normal representation $\lambda$ of $A$, the set $\lambda(S)$ contains a nonzero element of finite trace (Lemma 1). For each $x \in S$, let $I(x)$ be the ideal generated by $x$, and let $T(x)$ be the set of all bitraces $s$ on $I(x) \times I(x)$ such that $s(x, x)=1$. Let $T=T_{x}$ be the set of all bitraces $s$ in $T(x)$ such that $\lambda_{s}$ is a factor representation. The set $T_{x}$ is a Borel subset of the polonais space $T(x)$ and thus $T_{x}$ is standard (Theorem 3 and its proof). For $s \in T$, let $f=f_{s}$ denote the positive functional on $A$ given by $f(y)=s(y x, x)$ for $y \in A$. We note that 
$f$ is a state. Indeed, the property (v) of bitraces shows that the $\lim \lambda_{f}\left(u_{n}\right)=1$ in the strong topology on $H_{f}$ where $\left\{u_{n}\right\}$ is an increasing approximate identity in the positive part of the unit sphere of $A$. (The last statement, incidentally, shows the equivalence of the definition of bitraces used in this note and the definition used by Guichardet $[9, I, \S 1$, no. 1].) The canonical representation $\lambda=\lambda_{f}$ induced by $f$ is quasi-equivalent to $\lambda_{s}$. Because $\lambda_{s}$ is a factor representation, it is sufficient to show that $\lambda$ is equivalent to a subrepresentation of $\lambda_{s}[6,5.3 .5]$. For $x_{i}, y_{i}(1 \leqslant i \leqslant m)$ in $A$, the relation

$$
\begin{aligned}
\left(\sum L_{f}\left(x_{i}\right), \sum L_{f}\left(y_{i}\right)\right) & =\sum_{i, j}\left(L_{f}\left(x_{i}\right), L_{f}\left(y_{j}\right)\right) \\
& =\sum_{i, j} f\left(y_{j}^{*} x_{i}\right)=\left(\sum \Lambda_{s}\left(x_{i} x\right), \sum \Lambda_{s}\left(y_{i} x\right)\right)
\end{aligned}
$$

implies the existence of an isometric isomorphism $u$ of $H_{f}$ onto the closed subspace $H_{s}^{\prime}=\operatorname{clos} \lambda_{s}(A) \Lambda_{s}(x)$ of $H_{s}$. The projection $e^{\prime}$ of $H_{s}$ onto $H_{s}^{\prime}$ lies in the commutant $\lambda_{s}(A)^{\prime}$ of $\lambda_{s}(A)^{\prime \prime}$ and satisfies the relation

$$
u^{-1} \lambda(y) u=\lambda_{s}(y) e^{\prime}
$$

for every $y \in A$. This proves that $\lambda \sim e^{\prime} \lambda_{s}$, and consequently, that $\lambda \sim \lambda_{s}$.

Now let $\Phi=\Phi_{x}$ be the map of $T$ into $F(A)$ given by $\Phi(s)=f_{s}$. It is clear that $\Phi$ is continuous. We have that $\Phi$ is one-one; in fact, by the proof of Theorem 3, we have more: If $\Phi(r) \sim \Phi(s)$, then $\lambda_{r} \sim \lambda_{s}$ and consequently $r=s$. Hence, we get that $\Phi$ is a one-one Borel map of the standard Borel space $T$ into the standard Borel space $F(A)$. This means that $\Phi(T)$ is a Borel subset of $F(A)$ [1, Lemma 2.5]. It is clear that $\Phi(T)$ meets each quasi-equivalence class of $F(A)$ in at most one point.

Let $\left\{x_{i}\right\}$ be an enumeration of $S$. Let $\Phi_{i}=\Phi_{x_{i}}$ and let $T_{x_{i}}=T_{i}$. We have that $\psi_{1}\left(\Phi_{i}\left(T_{i}\right)\right)=Y_{i}$ is a Borel set in $\widetilde{A}[11$, Proposition 10]. Since the saturation $Z_{i}$ of $\Phi_{i}\left(T_{i}\right)$ can be expressed as $Z_{i}=\psi_{1}^{-1}\left(Y_{i}\right)$, we conclude that $Z_{i}$ is a Borel set in $F(A)$. We define the Borel sets $\left\{X_{i} \mid i=1,2, \cdots\right\}$ in $F(A)$ by

$$
X_{1}=\Phi_{1}\left(T_{1}\right) \text { and } X_{i}=\Phi_{i}\left(T_{i}\right)-\bigcup_{j<i} Z_{j}
$$

for $i>1$. Then $X_{0}=\bigcup X_{i}$ is a Borel subset of $X$ and is a transversal for $X$. We first verify that two quasi-equivalent factor states $f$ and $g$ in $X_{0}$ are equal. Suppose that $f \in X_{i}$ and $g \in X_{j}$. Since $Z_{i}$ and $Z_{j}$ are saturated and $f$ and $g$ lie in $Z_{i}$ and $Z_{j}$ respectively, we must conclude that $i=j$. However, the set $X_{i}$ is contained in the set $\Phi_{i}\left(T_{i}\right)$ which meets each quasi-equivalence 
class in at most one point. Hence we have that $f=g$.

Now we prove that each $f \in X$ is in the saturation of $X_{0}$. By Theorem 3 , there is an $s \in T_{i}$ for some positive integer $i$ such that $\psi\left(\theta_{x_{i}}(s)\right)=\left[\lambda_{f}\right]$. (Here we are employing the notation of Theorem 3.) We may assume that this $i$ is the smallest such integer for which such an $s$ exists. Then we have that $\lambda_{f} \sim \theta_{x_{i}}(s) \sim \lambda_{s} \sim \lambda_{f_{s}}$, and consequently, that $f \sim f_{s}=\Phi_{i}(s)$. For every $j<i$, we get that $f_{s} \notin Z_{j}$; otherwise, there is an $r \in T_{j}$ such that $f_{r} \sim f_{s} \sim f$ or equivalently such that $\psi\left(\theta_{x_{j}}(r)\right)=\left[\lambda_{f}\right]$. This proves that $f$ is in the saturation of $X_{0}$. Q.E.D.

\section{BIBLIOGRAPHY}

1. L. Auslander and C. C. Moore, Unitary representations of solvable Lie groups, Mem. Amer. Math. Soc. No. 62, 1966. MR 34 \#7723.

2. N. Bourbaki, Eléments de mathématique. VIII. Part I: Les structures fondamentales de l'analyse. Livre III: Topologie générale, Chap. 9, Actualités Sci. Indust., no. 1045, Hermann, Paris, 1948. MR 10, 260.

3. E. B. Davies, Decomposition of traces on separable C*-algebras, Quart. J. Math Oxford Ser. (2) 20 (1969), 97-111. MR 39 \#1984.

4. J. Dixmier, Traces sur les $C^{*}$-algèbres, Ann. Inst. Fourier (Grenoble) 13 (1963), fasc. 1, 219-262. MR 26 \#6807; erratum, 30, p. 1203.

5. —, Traces sur les $C^{*}$-algèbres. II, Bull. Sci. Math. (2) 88 (1964), 39-57.

MR 31 \#6132.

6. - Les $C^{*}$-algèbres et leurs représentations, Cahiers Scientifiques, Fasc. XXIX, Gauthier-Villars, Paris, 1964. MR 30 \#1404. 1969.

7. - Les algèbres d'opérateurs dans l'espace hilbertien, Gauthier-Villars, Paris,

8. J. A. Ernest, A decomposition theory for unitary representations of locally compact groups, Trans. Amer. Math. Soc. 104 (1962), 252-277. MR 25 \#3383.

9. A. Guichardet, Caractères des algèbres de Banach involutives, Ann. Inst. Fourier (Grenoble) 13 (1963), fasc. 1, 1-81. MR 26 \#5437; erratum, 30, p. 1203.

10. H. Halpern, Commutators in properly infinite von Neumann algebras, Trans. Amer. Math. Soc. 139 (1969), 53-73. MR 40 \#4773.

11. - Mackey Borel structure for the quasi-dual of a separable $C^{*}$-algebra, Canad. J. Math. 26 (1974), 621-628.

12. G. W. Mackey, Borel structure in groups and their duals, Trans. Amer. Math. Soc. 85 (1957), 134-165. MR 19, 752.

13. G. K. Pedersen, $C^{*}$-integrals, an approach to non-commutative measure theory, Lecture notes, Philadelphia, 1972.

14. F. Perdrizet, Topologie et traces sur les $C^{*}$-algèbres. Bull. Soc. Math. France, 99 (1971), 193-239. MR 45 \#2486.

15. S. Sakai, $C^{*}$-algebras and $W^{*}$-algebras, Springer-Verlag, New York, 1971. OHIO 45221

DEPARTMENT OF MATHEMATICS, UNIVERSITY OF CINCINNATI, CINCINNATI, 